\section{Intracranial hemorrhage in patient treated with rivaroxaban}

\author{
Michelle Molina, Virany H. Hillard, \\ Robert Fekete
}

Department of Neurology, New York Medical College, Valhalla, NY, USA

\begin{abstract}
Rivaroxaban is an oral factor Xa inhibitor used for stroke prevention in atrial fibrillation. There are currently no evidence-based guidelines for the treatment of hemorrhagic side effects of factor Xa inhibitors. We report a case of a thalamic hemorrhage in an 84 year-old right-handed female on rivaroxaban for treatment of atrial fibrillation. The patient had fallen down steps and became unresponsive. She was found to have diffuse scattered acute subarachnoid hemorrhage as well as intraventricular hemorrhage. Neurosurgical intervention was not required in this case, but controversy over decision making to pursue pro-coagulant therapy in the setting of worsening hemorrhage requiring emergent surgery is discussed.
\end{abstract}

\section{Introduction}

Rivaroxaban is an oral factor Xa inhibitor used for stroke prevention in the setting of atrial fibrillation. There are currently no evidence-based guidelines for the treatment of hemorrhagic complications of factor Xa inhibitors. We report the second case of an intracranial hemorrhage in a patient on rivaroxaban for treatment of atrial fibrillation.

\section{Case Report}

This is an 84 year-old right-handed female with a past medical history of atrial fibrillation on rivaroxaban $20 \mathrm{mg}$ daily, Alzheimer's dementia, hypertension, diabetes, and chronic obstructive pulmonary disease. The patient had fallen down 6 steps at home as witnessed by her husband. Her last rivaroxaban dose was about 8 hours prior to the fall. She became unresponsive 2 hours after the fall without tonic or clonic activity and was intubated in the field for airway protection. In addition she received 1 gram of levatiracetam and $1 \mathrm{mg}$ of lorazepam.

Physical examination was significant for left forehead and right lower extremity ecchymoses. On computed tomography (CT) scan at time of presentation in the emergency room, she was found to have diffuse scattered acute subarachnoid hemorrhage and intraventricular hemorrhage (Figure 1). Repeat head CT less than 24 hours after initial event showed stable acute subarachnoid hemorrhage and intraventricular hemorrhage. Laboratory evaluation at admission showed prothrombin time of 11.7 seconds, activated partial thromboplastin time of 24.7 seconds, and platelet count of 157,000 per $\mathrm{mm}^{3}$. At 3 hours post event, she received four units of fresh frozen plasma and $10 \mathrm{mg}$ of Vitamin K slow intravenous push. The patient's hospitalization was complicated by intubation, pneumonia, and gastrostomy tube placement. Rivaroxaban was held during the hospitalization. Levetiracetam was initiated for seizure prophylaxis and was tapered off after two weeks. She was able to recover to her prior neurological baseline. Warfarin anti-coagulation was started 2-3 weeks after onset of the hemorrhage.

\section{Discussion}

In this case we demonstrate an acute posttraumatic subarachnoid and intraventricular hemorrhage in the setting of use of rivaroxaban for the treatment of atrial fibrillation. Clinical details of the current case as well as those of a previously reported case of hemorrhagic conversion of ischemic stroke while on rivaroxaban treatment for atrial fibrillation are
Correspondence: Robert Fekete, Department of Neurology, New York Medical College, Munger Pavilion, 4th Floor, 40 Sunshine Cottage Road, Valhalla, NY 10595, USA.

Tel. +1.914.594.4293 - Fax: +1.914.594.4295.

E-mail: robertfekete@hotmail.com

Key words: rivaroxaban, Factor Xa inhibitor.

Contributions: MM, writing of the first draft, obtaining consent; VHH and RF, initial planning, obtaining imaging, revision and critique.

Conflict of interests: RF served as consultant to Lundbeck, LLC., and Teva Neuroscience, Inc.; he received honoraria from Medlink, Inc. Other authors have nothing to disclose.

Received for publication: 2 January 2014.

Revision received: 12 February 2014.

Accepted for publication: 24 March 2014.

This work is licensed under a Creative Commons Attribution NonCommercial 3.0 License (CC BYNC 3.0).

(C) Copyright M. Molina et al., 2014

Licensee PAGEPress, Italy

Hematology Reports 2014; 6:5283

doi:10.4081/hr.2014.5283

presented in Table $1 .^{1}$ In both cases, neurosurgical intervention was not necessary due to mild evolution which did not significantly increase mass effect.

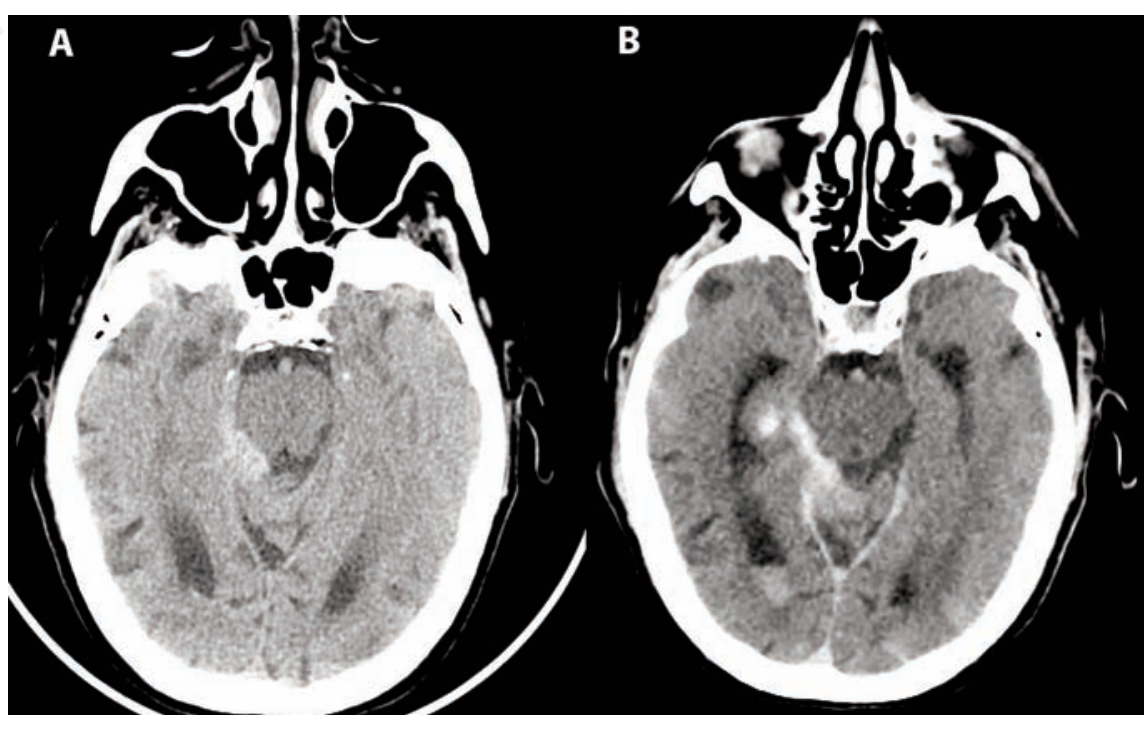

Figure 1. Diffuse subarachnoid hemorrhage affecting cortical sulci, right quadrigeminal plate, interpeduncular fossa, and intraventricular hemorrhage at initial presentation (A) and with mild interval increase 8 hours later (B). 
Table 1. Clinical details of known rivaroxaban intracranial hemorrhage cases.

\begin{tabular}{|c|c|c|c|c|c|c|}
\hline Age & Sex & Rivaroxaban dose & Indication & Length of treatment & Hospital treatment & Diagnosis \\
\hline 84 & $\mathrm{~F}$ & 20 mg daily & Atrial fibrillation & One year & Fresh Frozen Plasma & $\begin{array}{l}\text { Subarachnoid and } \\
\text { intraventricular hemorrhage }\end{array}$ \\
\hline 60 & M & 15 mg daily & Atrial fibrillation & 16 days & $\begin{array}{c}\text { Fresh Frozen Plasma + } \\
\text { Vitamin K }\end{array}$ & $\begin{array}{l}\text { Hemorrhagic conversion } \\
\text { of ischemic stroke }\end{array}$ \\
\hline
\end{tabular}

While Fresh Frozen Plasma treatment was provided, reversal of rivaroxaban's effect was mostly likely achieved via clearance of rivaroxaban. In retrospect, the administration of Vitamin $\mathrm{K}$ was not appropriate for this clinical setting. There was no elevation in prothrombin time and activated partial thromboplastin time to suggest accumulation of rivaroxaban. A limitation of this report is that laboratory testing of rivaroxaban concentration is not available at our facility.

Unfortunately, there is no clinically proven method of emergently reversing rixaroxaban's effect in the setting of acute hemorrhage. In a rabbit model, Godier et al. report that recombinant activated factor VII and prothrombin complex concentrate only partially improved the laboratory parameters, but did not reverse rivaroxaban induced-bleeding. ${ }^{2}$ The working group on perioperative hemosta- sis advises if possible waiting until clearance of rivaroxaban occurs, ${ }^{3}$ but this is not an option in in neurosurgical emergencies with possible herniation risk. The working group discusses using activated or non-activated prothrombin complex concentrates, which unfortunately have been associated with thrombotic complications, which becomes a concern especially in patients with existing atrial fibrillation.

\section{Conclusions}

The lack of reliable reversal agents for rivaroxaban should be considered when initiating pharmacotherapy in patients with high fall risk.

\section{References}

1. Fekete R, Jeevan D, Marks S, Hillard VH. Hemorrhagic transformation of ischemic stroke in patient treated with rivaroxaban. J Hematol 2013;2:48-50.

2. Godier A, Miclot A, Le Bonniec B, et al. Evaluation of prothrombin complex concentrate and recombinant activated factor VII to reverse rivaroxaban in a rabbit model. Anesthesiology 2012;116:94-102.

3. Pernod G, Albaladejo P, Godier A, et al. Management of major bleeding complications and emergency surgery in patients on long term treatment with direct oral anticoagulants, thrombin or factor-Xa inhibitors: proposals of the Working Group on Perioperative Haemostasis. Arch Cardiovasc Dis 2013;106:382-93. 